\title{
BMJ Open Does exercise impact gut microbiota composition in men receiving androgen deprivation therapy for prostate cancer? A single-blinded, two-armed, randomised controlled trial
}

\author{
Robert U Newton, ${ }^{\oplus 1,2,3}$ Claus T Christophersen, ${ }^{\circledR 3,4}$ Ciaran M Fairman, ${ }^{\oplus 1,3}$ \\ Nicolas H Hart, ${ }^{\oplus 1,3,5}$ Dennis R Taaffe, ${ }^{\oplus 1,2,3}$ David Broadhurst, ${ }^{\oplus 6,7}$ \\ Amanda Devine, ${ }^{3,7}$ Raphael Chee, ${ }^{1,3,8}$ Colin I Tang, ${ }^{9}$ Nigel Spry, ${ }^{\oplus 1,3,8}$ \\ Daniel A Galvão 1,3
}

To cite: Newton RU, Christophersen CT, Fairman CM, et al. Does exercise impact gut microbiota composition in men receiving androgen deprivation therapy for prostate cancer? A single-blinded, two-armed, randomised controlled trial. BMJ Open 2019;9:e024872. doi:10.1136/ bmjopen-2018-024872

- Prepublication history for this paper is available online To view these files, please visit the journal online (http://dx.doi. org/10.1136/bmjopen-2018024872).

Received 19 June 2018 Revised 15 January 2019 Accepted 26 February 2019

Check for updates

(C) Author(s) (or their employer(s)) 2019. Re-use permitted under CC BY-NC. No commercial re-use. See rights and permissions. Published by BMJ.

For numbered affiliations see end of article.

Correspondence to Professor Robert U Newton; r.newton@ecu.edu.au

\section{ABSTRACT}

Introduction A potential link exists between prostate cancer (PCa) disease and treatment and increased inflammatory levels from gut dysbiosis. This study aims to examine if exercise favourably alters gut microbiota in men receiving androgen deprivation therapy (ADT) for PCa. Specifically, this study will explore whether: (1) exercise improves the composition of gut microbiota and increases the abundance of bacteria associated with health promotion and (2) whether gut health correlates with favourable inflammatory status, bowel function, continence and nausea among patients participating in the exercise intervention.

Methods and analysis A single-blinded, two-armed, randomised controlled trial will explore the influence of a 3-month exercise programme (3 days/week) for men with high-risk localised PCa receiving ADT. Sixty patients will be randomly assigned to either exercise intervention or usual care. The primary endpoint (gut health and function assessed via feacal samples) and secondary endpoints (self-reported quality of life via standardised questionnaires, blood biomarkers, body composition and physical fitness) will be measured at baseline and following the intervention. A variety of statistical methods will be used to understand the covariance between microbial diversity and metabolomics profile across time and intervention. An intention-to-treat approach will be utilised for the analyses with multiple imputations followed by a secondary sensitivity analysis to ensure data robustness using a complete cases approach.

Ethics and dissemination Ethics approval was obtained from the Human Research Ethics Committee of Edith Cowan University (ID: 19827 NEWTON). Findings will be reported in peer-reviewed publications and scientific conferences in addition to working with national support groups to translate findings for the broader community. If exercise is shown to result in favourable changes in gut microbial diversity, composition and metabolic profile, and reduce gastrointestinal complications in PCa patients receiving ADT, this study will form the basis of a future phase III trial.
Strengths and limitations of this study

- This trial comprises a comprehensive analysis of gut microbiome in individuals with prostate cancer, addressing a significant and increasingly emergent issue in this area.

- A two-armed, randomised controlled trial design to examine proof of concept and provide effect sizes to design subsequent appropriately powered trials.

Lack of follow-up past 13 weeks prohibits any assessment of sustainability of effects.

- The inclusion criteria prevent generalisability to the other types of cancer.

Diet and physical activity (outside of the intervention) will not be tracked and are limitations of the current design.

Trial registration number ANZCTR12618000280202.

\section{INTRODUCTION}

Gut microbiota play a fundamental role in protection from pathogens, in the induction and function of the immune system and in the promotion of digestion and absorption of dietary nutrients for energy production. ${ }^{12}$ Indeed, perturbations of the composition and function of gut microbiota can cause gastrointestinal (GI) complications, and contribute to the pathogenesis of metabolism and disease processes such as inflammation, infection and tumours. ${ }^{3-6}$

Androgen deprivation therapy (ADT) is extensively used as a neoadjuvant, adjuvant and/or standalone treatment for men with prostate cancer $(\mathrm{PCa}) ;{ }^{6}$ however, survivors are burdened with persistent adverse effects of ADT including GI complications..$^{7-11}$ 
Despite a paucity of research into the mechanisms associated with GI diseases in PCa survivors, it is hypothesised that a dysbiotic composition of the gut microbiome may mediate negative outcomes of GI complications associated with PCa. ${ }^{12-15}$ Indeed, results from murine models demonstrate that castration and antiandrogens can affect the composition of gut microbiota. ${ }^{16} 17$ The complex, dynamic interaction between sex hormones and gut microbiome is still poorly understood. ${ }^{18}$ Nevertheless, Markle $e a^{19}$ demonstrated that elevated levels of testosterone in female mice results in favourable changes in microbiota, metabolomics and inflammation, lending potential support for the role of ADT in adverse changes to gut microbiota. ${ }^{190}$ To address gut dysbiosis, especially for those with cancer, recent interventions have focused on the use of probiotics or prebiotics to alleviate symptoms, ${ }^{2}$ indicating that the manipulation of gut microbiota could be a viable therapeutic strategy for men with PCa. ${ }^{21} 22$

Perhaps unsurprisingly, emerging evidence indicates that exercise may exert a positive effect on gut microbial composition. ${ }^{23}$ In non-cancer cohorts, a link between exercise and gut microbial diversity, composition and beneficial metabolite production has been illustrated. ${ }^{24} 25$ Of note, the ratio of Firmicutes to Bacteroidetes phyla is of particular importance, with obese individuals having a higher Firmicutes to Bacteroidetes ratio which is indicative of poorer gut health. ${ }^{26}{ }^{27}$ Importantly, it has been consistently demonstrated that men on ADT experience considerable unfavourable shifts in body composition, indicated by a significant loss of lean body mass coupled with an increase in fat mass. ${ }^{28-30}$ Consequently, men on ADT may be at a heightened risk for this altered ratio of Firmicutes to Bacteroidetes. The shift in relative abundances of these phyla is suggested to result in an increased ability to harvest energy from food and promote low-grade inflammation. ${ }^{31}$ Whereas, a microbiota in homeostasis and the presence of Lactobacillus and Bifidobacterium can exert anti-inflammatory effects at local and systemic levels, indicating a potential link between the gut microbiota health and inflammatory status. ${ }^{32}$

Metabolites are molecules that have critical roles in cellular metabolism, energy production and storage, apoptosis and signal transduction, and are affected by diet, exercise and gut microbiota among others. Indeed, the metabolomic profile has been suggested to be indicative of disease state in cancer and identified as a potential biomarker of the development and progression of PCa. ${ }^{33}$ Consequently, an exploration of the relationships between changes in gut microbiota composition, metabolomic profile, inflammatory status and bowel function could have important clinical implications through enhancing positive exercise-induced effects on these outcomes through the manipulation of the composition of gut microbiota. Although the potential for exercise to modulate gut microbiota is promising, a paucity of research in the area exists, particularly in individuals at risk for gut dysbiosis. As such, no prior investigation has been conducted to determine whether gut microbiota composition is influenced by exercise in PCa survivors. ${ }^{34}$

The aim of this exploratory study is to determine the effect of exercise medicine on gut microbiota and its metabolome in men receiving ADT for PCa. Specifically, we will explore whether: (1) exercise improves composition of the gut microbiota and increases abundance of health-promoting bacterial species, thereby driving an advantageous faecal metabolomic profile and (2) improved gut health correlates with favourable inflammatory status, bowel function, continence, nausea and appetite among patients participating in the exercise intervention. It is hypothesised that the exercise programme will result in favourable changes in the composition of gut microbiota, and that these changes will be correlated with improvements in inflammatory status, bowel function and other GI issues. This study will serve to provide a better understanding of whether the gut microbiota and its metabolites can be modulated by exercise as a potential therapeutic target that can be offered for PCa patients on ADT.

\section{METHODS}

\section{Study design}

This is a single-blinded (investigators blinded to group allocation), randomised controlled trial, designed to measure the impact of a supervised exercise medicine intervention on gut microbiota and its metabolome of PCa patients receiving ADT. An 'exercise' group will receive a 12-week supervised exercise programme comprising aerobic and resistance exercise undertaken three times per week. A 'usual care' group will continue to receive usual medical care during the trial. The usual care group will be offered an exercise programme following their control period to minimise study contamination and to reduce patient withdrawal and loss to follow-up.

\section{Participants}

Sixty men $(\mathrm{n}=60)$ with high-risk localised PCa, defined by the National Comprehensive Cancer Network as T3a disease, Gleason $\geq 8$ or prostate- specific antigen (PSA) $\geq 20,{ }^{35}$ who are currently on ADT (and expected to remain on ADT for the next 3 months) are eligible to enrol in this study and will be randomised to exercise or usual care. ADT can be achieved with either luteinising hormone-releasing hormone (LHRH) agonists or LHRH antagonists, or a combination of the two. It is also acceptable for patients to receive no $>4$ weeks of antiandrogen at the initiation of their ADT to prevent flare from testosterone surge. Patients must be on continuous ADT during the study period.

Participants will be excluded if they have any nodal, visceral or bone metastases (N1 or M1 disease); are being treated for other cancers; previously received or currently receiving chemotherapy; currently receiving radiation therapy or any non-approved experimental therapies; and/or previously had any prior GI surgery. Patients will 
also be excluded if they have taken antibiotics, prebiotics, probiotics, nutritional or ergogenic compliments or supplements within 3 months prior to study enrolment; have recently had a prostatectomy, orchiectomy or received radiation therapy within 12 months prior to study enrolment; have any physical, mental or other contraindications to exercise; and/or cannot (or have difficulty) understanding English. Participants will be excluded if they currently engage in regular, structured physical activity (aerobic or resistance training for two or more times per week in the previous 3 months). All participants must receive medical clearance from their managing physician to be eligible to enrol. Additionally, all participants will be asked to provide informed consent prior to the start of any study activities.

\section{Recruitment}

Men with localised or locally advanced PCa, who are currently receiving $\mathrm{ADT}$, will be recruited by invitation of their attending specialist (ie, urologist or radiation oncologist), who will provide clinically eligible patients with a study information sheet and referral to the study coordinator. If patients are interested in participation, and their eligibility is confirmed, they will receive an informed consent document to read and sign in the presence of a study investigator and/or clinical research coordinator prior to completing baseline measures and randomisation. Figure 1 outlines the process of referrals, recruitment and enrolment in the trial.

\section{Randomisation}

PCa patients will be randomly allocated in a ratio of 1:1 to two study arms: exercise or usual care, stratified by age ( $\leq 60$ and $>60$ years), time on ADT ( $\leq 6$ and $>6$ months) and prior prostatectomy or radiation therapy (Yes and No) for approximate balance between groups in order to mitigate confounding factors pertaining to variations due to ageing (ie, differences in physical function, sarcopenia, osteopenia and osteoporosis) and to account for variations in treatment. All patients will be required to be on ADT prior to, and during the study, as per standard of care for this patient population. A research officer with no patient contact will be responsible for the randomisation of patients into groups using a computer-generated random assignment programme. Study investigators and the exercise physiologists conducting testing procedures will be blinded to group allocation. Only exercise physiologists who are not part of the research team will be permitted to deliver the exercise intervention to participants in order to maintain integrity of the blinding process.

\section{Measurements}

Table 1 outlines the primary and secondary endpoints that will be undertaken at baseline (week 0) and postintervention (week 13). All procedures and assessment tools have proven validity and reliability and are used widely throughout clinical research.
Referral by specialists in Western Australia
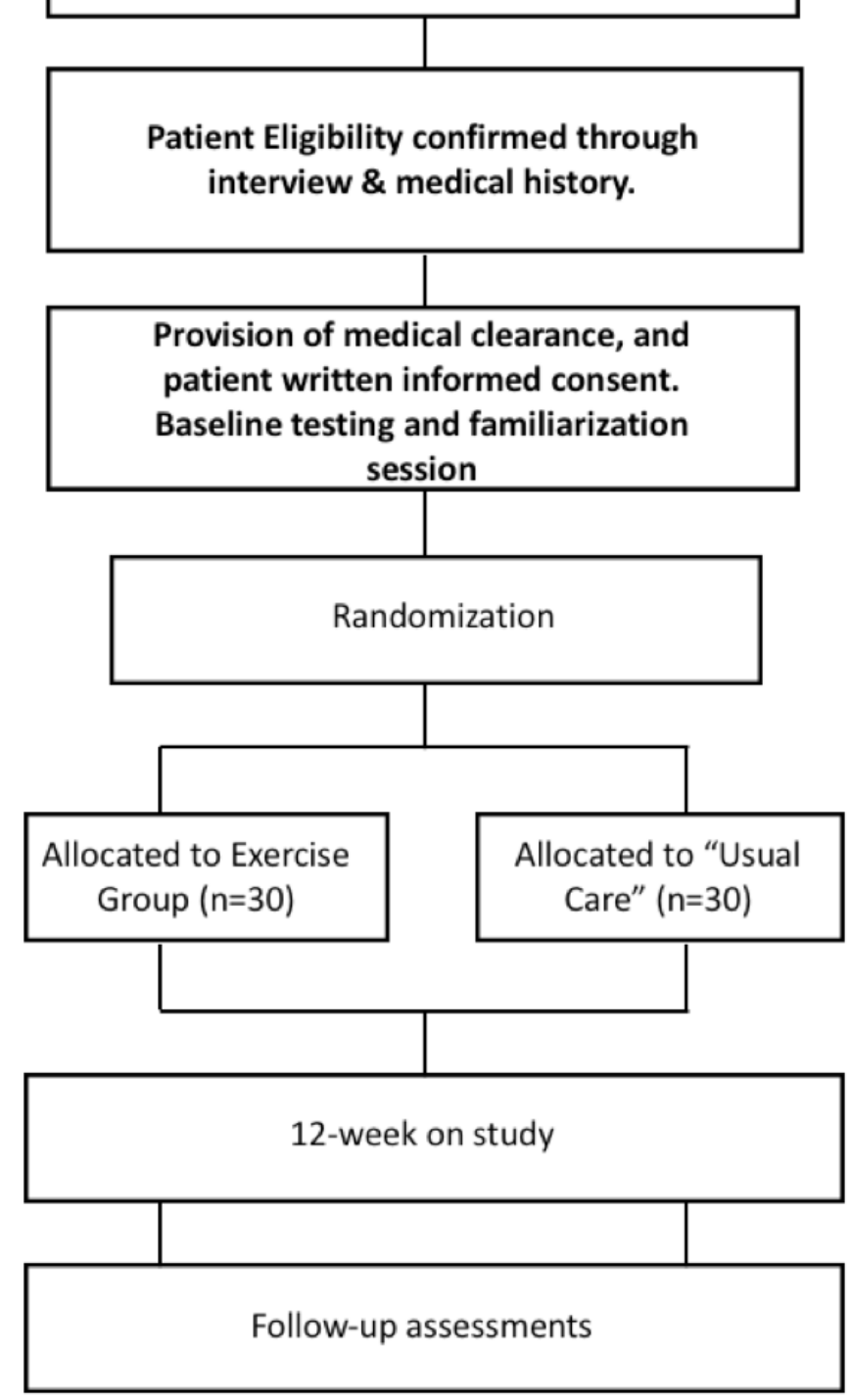

Figure 1 Consolidated Standards of Reporting Trials diagram.

\section{Primary endpoint}

Gut health and dysbiosis

Gut health will be assessed through the provision of a 24-hour collection of stool sample(s), subsequently examined and analysed for microbial composition (using bacterial DNA), bacterial metabolites and for faecal calprotectin. Briefly, participants will be provided with a 24-hour collection kit for collection of all bowel movements within that time. The kit includes a high-performance cooler bag (Techni Ice), two gel ice packs (Techni Ice), collection bags, Bristol stool chart to classify stool and instructions. The cooler bag has been tested in our laboratory to maintain temperature at $4^{\circ} \mathrm{C}$ for $24-36$ hours, and $4^{\circ} \mathrm{C}$ has been shown to not significantly alter faecal microbiota diversity or composition. ${ }^{36}$ In addition, we have also showed that there is no significant difference in faecal short-chain fatty acid (SCFA) levels between cool temperature storage and 
Table 1 Schedule of assessments at baseline and postintervention

\begin{tabular}{lll}
\hline Measures & Baseline & Postintervention \\
\hline Medical history & $\mathrm{x}$ & \\
Gut health (24-hour stool sample) & $\mathrm{x}$ & $\mathrm{x}$ \\
\hline Dietary behaviour (DQES) & $\mathrm{x}$ & $\mathrm{x}$ \\
Blood biomarkers & $\mathrm{x}$ & $\mathrm{x}$ \\
Cancer-specific quality of life & & \\
\multicolumn{1}{c}{ EORTC-QLQ-C30 } & $\mathrm{x}$ & $\mathrm{x}$ \\
\hline EORTC-PR-25 & $\mathrm{x}$ & $\mathrm{x}$ \\
Body composition (DXA) & $\mathrm{x}$ & $\mathrm{x}$ \\
\hline Muscle strength (1RM) & $\mathrm{x}$ & $\mathrm{x}$ \\
\hline Aerobic fitness (400 m) & $\mathrm{x}$ & $\mathrm{x}$ \\
\hline Physical function tests & $\mathrm{x}$ & $\mathrm{x}$ \\
\hline
\end{tabular}

DQES, Dietary Questionnaire for Epidemiological Studies; EORTCQLQ-C30, European Organisation for Research and Treatment of Cancer Quality of Life Questionnaire Core 30; EORTC-PR-25; EORTC-Prostate Cancer Module 25; 1RM, one-repetition maximum.

immediate freezing of the stool sample at $-20^{\circ} \mathrm{C}$ (data not shown). Samples will be returned to the laboratory within 12-24hours following collection and stored at $-80^{\circ} \mathrm{C}$ in a biomedical freezer (Forma 88700 ULT; Thermo Fisher Scientific, Waltham, Massachusetts, USA) until they are homogenised (combined if $>1$ sample over the 24 hours) and partitioned into aliquots for analysis.

To extract microbial DNA, samples will be lysed using both mechanical and enzymatic breakdown of the bacterial cell membranes and the DNA extracted using the QIAamp PowerFecal DNA kit (Qiagen; Hilden, Germany). Microbiome signatures will be generated using the Illumina MiSeq platform using barcoded primer, V4 (515-806). Quality control samples are included in the analysis from sample collection to sequence analysis and all samples will be individually screened for PCR efficiency prior to building sequence libraries. For building the sequence libraries, a PCR-free ligation protocol will be deployed with sample barcodes that are never reused in our laboratory. Samples will be sequenced to a depth of minimum 30000 reads, which is sufficient to identify microbes to a genus/species level. Quantitative polymerase chain reaction ( $q$ PCR) will be used to verify shifts observed in the sequence data and will therefore be determined postanalysis of the $16 \mathrm{~S}$ rRNA gene data. This will provide a detailed picture of longitudinal changes in gut microbial community structure with treatment and following exercise.

Sequence read quality will be initially assessed with quality control software (FASTQC, https://www.bioinformatics.babraham.ac.uk/projects/fastqc/) before demultiplexing and preprocessing by Shi $7^{37}$ and/or GHAPv2. Clutadapt will be used for removal of all non-biological sequences. $^{38}$ DADA2 is then used for quality filtering, error correction, Amplicon Sequence Variance (ASV) picking. ${ }^{39}$ A trained naïve Bayes classifier then assigns ASVs to genus/species against a curated database of microbial reference sequences such as the $\mathrm{RDP}^{40}$ or SILVA.

The faecal metabolome contains over 6000 known endogenous and exogenous metabolites, comprised of many classes, each with unique chemical properties; thus, no single analytical platform can be used to measure the entire metabolome in a single assay. Untargeted metabolite profiles ( $>2000$ metabolite features) will be measured in faecal samples using high-throughput mass-spectrometry technologies consisting of two complementary analytical platforms in six modes of operation: gas chromatography high resolution mass spectrometry (GC-HRMS) with electronc impact (EI) and chemical ionization (CI) modes; and liquid chromatography high resolution mass spectrometry (LC-HRMS) (reverse phase and hydrophilic interaction (HILIC) chromatography with both positive and negative ionisation modes). Resulting data will provide a comprehensive systems overview of metabolome disruption of the gut microbiota due to PCa and $\mathrm{ADT}$, as well as alterations resulting from the exercise intervention. All analyses will be performed at the Centre for Integrative Metabolomics \& Computational Biology (CiMCB; Edith Cowan University, Perth, Australia). Additional targeted analysis methods will be used for accurate quantification of SCFAs; formate, acetate, propionate, butyrate, isobutyrate, valerate and isovalerate metabolites. Faecal calprotectin will also be measured as a biomarker of inflammation to monitor gut inflammation. ${ }^{41}$

\section{Secondary endpoints}

\section{Dietary behaviour}

Habitual dietary intake will be assessed using the Dietary Questionnaire for Epidemiological Studies (DQES V.2.0; Cancer Council of Victoria, Melbourne, Australia) which is a modified food frequency questionnaire relevant to cancer populations. A research assistant will supervise the completion of the DQES and use food models and charts, metric cups and spoons to increase accuracy. All participants will be instructed to maintain their habitual dietary patterns throughout the study. DQES data will be collated and batch analysed at the conclusion of the study by the Nutritional Assessment Office (Cancer Council of Victoria). Dietary analysis will include food item frequency and a comprehensive nutrient intake analysis.

\section{Biomarkers}

Fasted blood samples will be collected and analysed commercially by an accredited National Association of Testing Authorities laboratory (Australian Clinical Labs, Perth, Australia) for glucose, high-sensitivity C-reactive protein, tumour necrosis factor-alpha, IL-6, cholesterol (total, high-density lipoprotein, low-density lipoprotein), testosterone and PSA. Additional serum (serum separator tube (SST), $8 \mathrm{~mL}$ ) and plasma (EDTA, $9 \mathrm{~mL}$ ) samples will be collected at baseline and postintervention visits by a certified phlebotomist, and will be processed and stored in 
a $-80^{\circ} \mathrm{C}$ biomedical freezer (Forma 88700 ULT; Thermo Fisher Scientific) for subsequent metabolomics analysis at the CiMCB. For participants that provide consent, the samples will be stored for a duration of 5 years. We will be pursuing other research studies in advanced cancer patients, and this deidentified blood sample data may be of use to gain further insights and comparisons. For participants who do not provide consent for future storage (but do provide consent for this study), we will destroy any leftover samples following the analysis required for this study (within 6 months from time of patient enrolment).

\section{Clinical data}

PCa participant medical history, including PCa diagnosis (ie, Gleason score, stage of cancer, months since diagnosis, treatment history and time on ADT), comorbidities (ie, hypertension, osteoarthritis and others), concomitant medications (ie, opioids, beta-blockers, antihypertensives, blood cholesterol and others) and other diagnosed chronic diseases (ie, osteoporosis, diabetes, cardiovascular disease and others) will be sourced from patient medical records. Further, information pertaining to any related history of bowel symptoms ${ }^{42}$ GI symptoms ${ }^{43}$ and other bowel-related conditions (Bristol Stool Chart) ${ }^{44}$ will also be obtained.

\section{Quality of life, bowel function, continence, nausea and appetite}

Cancer-specific quality of life indices will be measured using the European Organisation for Research and Treatment of Cancer Quality of Life Questionnaire Core 30, ${ }^{45}$ assessing five functional scales (physical, role, emotional, cognitive and social functioning), three symptom scales (fatigue, pain, nausea and vomiting), a global health scale and six single items (dyspnea, insomnia, appetite loss, constipation, diarrhoea and financial difficulty). PCa-specific quality of life indices will be measured using the EORTC-Prostate Cancer Module $25,{ }^{46}$ assessing urinary symptoms, bowel symptoms, treatment-related symptoms and sexual functioning.

\section{Anthropometric and body composition measures}

Stature will be recorded to the $\sim 1 \mathrm{~cm}$ using a wall-mounted stadiometer (Model 222, Seca, Hamburg, Germany), with body mass recorded to the $\sim 0.1 \mathrm{~kg}$ using an electronic scale (AE Adams CPW Plus-200, Adam Equipment, Connecticut, USA). Whole body fat percentage, fat mass and lean mass will be measured using Dual-energy X-ray Absorptiometry (DXA; Horizon A, Hologic, Marlborough, Massachusetts, USA), with whole-body and appendicular segmentations generated in accordance with Hart and colleagues. ${ }^{47}$

\section{Physical fitness}

A battery of standard tests will be used to assess physical fitness, including (1) muscle strength, using one-repetition maximum (1RM) tests for chest press, seated row and leg press exercises; (2) aerobic fitness, using the $400 \mathrm{~m}$ walk test and (3) physical function, using the timed up and go, $6 \mathrm{~m}$ walk test (normal, fast and backward) and repeated chair rise. $^{4849}$

\section{Exercise programme}

Patients assigned to the exercise arm will attend three clinic-based supervised exercise sessions each week for 12 weeks consisting of moderate-to-high intensity resistance and aerobic exercise (interval and continuous). Each session will take $\sim 60 \mathrm{~min}$, including warm-up and cool-down, and will be supervised by accredited exercise physiologists (Exercise and Sports Science Australia). The exercise programme is designed to provide optimal stimulus to the cardiorespiratory and neuromuscular systems while maximising safety, compliance and retention, thus will be tailored, progressive, periodised and autoregulated in collaboration with the patient (ie, adjusted based on the patient's presentation at each session).

Resistance exercise will be prescribed using repetition maximums (RM), where patients will be required to perform 6-8 different resistance exercises using major muscle groups, at 6-12 RM (the maximal weight that can be lifted $6-12$ times each set, equivalent to $\sim 60 \%-85 \%$ of 1RM) for 3-4 sets per exercise to achieve moderate-tohigh intensity and volume. Aerobic exercise will include 20-30 min of moderate-to-vigorous intensity activity (equating to $\sim 60 \%-85 \%$ of estimated maximum heart rate; or a rating of perceived exertion [RPE] between 6 and 8 using the BORG 10 point scale ${ }^{50}$ ) involving a variety of modes including walking, jogging, cycling or rowing, using treadmills and/or stationary ergometers. Aerobic intensity will be monitored using heart rate monitors (Polar M400, H10 Sensor, Polar Electro, NSW, Australia) and an RPE chart for assessment. Patients will also be encouraged to perform additional aerobic exercise (such as brisk walking), outside of their supervised exercise clinic visits, progressing towards accruing a volume of activity consistent with national guidelines for health (ie, $150 \mathrm{~min}$ of physical activity, or a further $\sim 60-90$ min self-managed). Participants will be provided log books to detail aerobic activity, which will be reviewed in the clinic each week. Flexibility exercises for all joints considered important for function and for all muscles engaged during the session will be provided during the cool-down phase of each exercise session.

\section{Statistical analysis}

These sample sizes $(n=30$ per group, totalling $n=60)$ have been chosen to determine a moderate standardised effect $(\mathrm{d}=0.8)$ for the primary and most secondary outcome measures (alpha $=0.05$ ).

A variety of statistical methods will be used to understand the covariance between microbial diversity and metabolomics profile across time and intervention, including using classical parametric and non-parametric univariate statistical methods (eg, repeated measure analysis of variance [ANOVA]), as well as multivariate projection models (such as principal components analyses and partial least squares discriminant analyses). The purpose of this initial stage of data analysis will be to uncover latent correlated biochemical structure in the data, mapping metabolite concentrations to metabolic pathways and ultimately to determine candidate metabolic signatures associated with 
dysregulation of the gut microbiome. The resulting metabolite signatures will be integrated with quantified SCFA measurements to contextualise the results. The metabolomic and metagenomic data will be integrated using latent structure projection techniques including OnPLS, ${ }^{51}$ Multiblock Component Analysis ${ }^{52}$ and Regularised Generalised Canonical Correlation Analysis, ${ }^{53}$ which extracts a minimal number of globally predictive orthogonal latent components that exhibit maximal covariance and correlation between data blocks. This will allow us to perform metabolite functional mapping, which will allow us to start unpacking the biochemical mechanism of microbiome/ exercise interaction.

Statistical analyses will also include descriptive characteristics, t-tests, effect size and two-way (group x time) repeated measures ANOVA (or analysis of covariance, adjusted for baseline values, time on ADT and medication change). For categorical variables, Pearson chi-square will be used. Data will be examined using an intention-to-treat approach with multiple imputations followed by a secondary sensitivity analysis to ensure data robustness using a complete cases approach. Data will be completely deidentified, and subsequently coded in order to note which group/interventiona patient received; without any ability to identify the patient in any way. Any identifiable documents will be destroyed. The deidentified information will be kept electronically only, in a password locked folder, on a password locked computer, accessible only by the team investigators. All statistical techniques employed in this study will be implemented using open source software ( $\mathrm{R}$ or Python), which will be made publicly available at the time of dissemination.

\section{Adverse events}

Individuals will be monitored for any adverse events during exercise testing and training and will be documented accordingly. The study team has extensive experience in delivering exercise specifically tailored to individuals with $\mathrm{PCa}$ in a safe and efficacious manner. The risks of adverse events are low in this study and a data monitoring/safety committee has not been appointed.

\section{Patient and public involvement}

The Exercise Medicine Research Institute (EMRI) has consumer representatives (cancer patients, survivors and their family members) who volunteer their time to provide input into research directions and study designs. Furthermore, the study clinicians (CIT, RC) consult within the major public and private hospitals in Perth, Western Australia, each with high PCa caseloads, thus have used patient priorities, patient experience and patient preferences to help inform the development of the research questions and outcome measures, while aiding the study protocol to engage participants in a respectful, ethical and impactful way.

\section{Ethics and dissemination}

Any amendments to the protocol will be submitted to and reviewed the HREC and trial registration will be updated accordingly. If exercise is proven to result in favourable changes in gut microbial diversity, composition and metabolic profile, and reduce GI complications (inflammation, constipation, diarrhoea and nausea) in PCa patients receiving $\mathrm{ADT}$, this study will form the basis of a future phase III trial. These outcomes will provide further validation of exercise medicine in this population and add to efforts aimed at changing clinical practice. To reach a maximum number of clinicians, practitioners, patients and scientists, the results of this study will be published in international, high-impact peer-reviewed clinical and academic journals. In addition, outcomes will be disseminated through national and international clinical, medical or academic conferences and oncology clinic and patient meetings. Finally, EMRI and the broader study team work closely with the Prostate Cancer Foundation of Australia (PCFA), their support groups and states offices. PCFA will assist in the translation and dissemination of the research findings to community members, and cancer support groups, while study participants will receive their individual results in addition to overall study findings.

\section{DISCUSSION}

PCa patients are burdened with persistent adverse effects of treatment, including GI complications. ${ }^{7-11}$ It has been hypothesised that the hormonal consequences of ADT may have an adverse effect on the composition of gut microbiota. ${ }^{20}$ Perturbations of the composition and function of gut microbiota can cause GI complications and contribute to the development and pathogenesis of metabolism and disease processes such as inflammation, infections and disease progression. ${ }^{14}$ Additionally, the metabolomic profile of PCa patients could be an important biomarker for disease state and progression. ${ }^{33}$ Consequently, an examination of therapeutic strategies to induce clinically meaningful improvements in the gut microbiome, metabolomic profile and inflammatory status is warranted.

Preliminary evidence indicates that exercise, independent of diet, may result in favourable changes in gut microbial diversity and composition, which has the potential to improve overall metabolic and health profile. ${ }^{54}$ While this research is promising, much of this area is in its infancy, with a substantial lack of evidence in a cancer context. Given the paucity of data, coupled with the potential negative health effects of gut dysbiosis in PCa, there is a clear need to directly evaluate the relationship between exercise and gut microbiota in this patient population. The protocol outlined in this paper will evaluate the effects of exercise on changes in the composition and diversity of gut microbiota and if these changes are correlated with favourable inflammatory status, bowel function, continence, nausea and appetite among patients participating in the exercise intervention.

Excess adiposity is associated with a heightened inflammatory state and gut dysbiosis. ${ }^{55}$ Exercise has been previously demonstrated to successfully attenuate and/or 
reverse the accumulation of fat mass experienced by $\mathrm{PCa}$ patients undergoing ADT. ${ }^{49}{ }^{56}$ Consequently, it is biologically plausible that improvements in lean body mass and overall body composition may mediate favourable changes in the composition of gut microbiota, inflammatory status and metabolomic profile. Moreover, gut dysbiosis has been associated with frailty in older adults. ${ }^{57-60}$ Though a causal relationship cannot be determined, improvements in muscle strength and physical function from exercise may contribute to improvements in the composition of gut microbiota in this population. Consequently, analysis of body composition and physical fitness will provide valuable information on any factors that may mediate changes in gut microbiome from exercise. ${ }^{60}$

\section{LIMITATIONS}

Despite the novel study design, several limitations should be acknowledged. Though a dietary intervention and/or more rigorous tracking throughout the study would be interesting, limitations in funding, resources and feasibility make it difficult to do this in the current study. An interesting aspect would be to examine the differential efficacy in androgen blockade, sex hormone conversion in the patient and subsequent exercise intervention effects. However, this is not possible in the current study and thus a limitation of the investigation is the ability to link androgen kinetics directly to the primary outcomes. Additionally, although this study will be one of the first to examine changes in gut microbiota with exercise in men with ADT, the lack of follow-up precludes an analysis of the sustainability of outcomes.

\section{CONCLUSIONS}

If demonstrated that this exercise intervention results in clinically meaningful improvements in gut health, the outcomes of this trial will provide innovative, new evidence that can inform the design of future phase III clinical trials to further elucidate the effects of exercise on gut health in patients with PCa. Further, any correlations between changes in the composition and diversity of gut microbiota and inflammatory status, and GI issues, may inform design of future research studies that further explore these relationships.

\footnotetext{
Author affiliations

${ }^{1}$ Exercise Medicine Research Institute, Edith Cowan University, Perth, Western Australia, Australia

${ }^{2}$ School of Human Movement and Nutrition Sciences, University of Queensland, Brisbane, Queensland, Australia

${ }^{3}$ School of Medical and Health Sciences, Edith Cowan University, Joondalup, Western Australia, Australia

${ }^{4}$ School of Molecular and Life Science, Curtin University - Perth City Campus, Perth, Western Australia, Australia

${ }^{5}$ Institute for Health Research, University of Notre Dame Australia, Perth, Western Australia, Australia

${ }^{6}$ School of Science, Edith Cowan University, Perth, Western Australia, Australia ${ }^{7}$ Centre for Integrative Metabolomics and Computational Biology, Edith Cowan University, Perth, Western Australia, Australia
}

${ }^{8}$ Department of Radiation Oncology, Genesis Cancer Care, Perth, Western Australia, Australia

${ }^{9}$ Department of Radiation Oncology, Sir Charles Gairdner Hospital, Perth, Western Australia, Australia

Acknowledgements The authors wish to thank Dr Lydia Hearn for her assistance with preparing the manuscript, and the consumer representatives who donated their time to review the study concept and provide input into the study protocol. The authors would also like to thank the many members of the community who donate to, and volunteer for, the Prostate Cancer Foundation of Australia which has funded this study.

Contributors All authors (RUN, CTC, NHH, DRT, DB, AD, RC, CMF, CIT, NS and DAG) contributed to the design and development of the study protocol. RUN, CTC, NHH, $\mathrm{DRT}, \mathrm{DB}, \mathrm{AD}, \mathrm{CMF}$ and DAG contributed to writing and editing of the manuscript. RC CIT and NS provided clinical input to the editing of the manuscript and will provide patient referrals to the study. All authors reviewed and approved the study protocol and have met the International Committee of Medical Journal Editors (ICMJE) recommendations.

Funding This study is funded by Prostate Cancer Foundation Australia (PCFA), New Concepts Grant scheme. NHH is supported by Cancer Council Western Australia Postdoctoral Fellowship. DRT and DB are supported by Professorial Research Fellowships of Edith Cowan University. CMF is supported by an NHMRC Centre of Research Excellence (Prostate Cancer Survivorship) Postdoctoral Fellowship.

Competing interests None declared.

Patient consent for publication Not required.

Ethics approval Ethics approval was obtained from the Human Research Ethics Committee (HREC) of Edith Cowan University (ID: 19827 NEWTON), with additional approval provided by the Radiation, Biosafety and Hazardous Substances Committee (RBHSC).

Provenance and peer review Not commissioned; externally peer reviewed.

Open access This is an open access article distributed in accordance with the Creative Commons Attribution Non Commercial (CC BY-NC 4.0) license, which permits others to distribute, remix, adapt, build upon this work non-commercially, and license their derivative works on different terms, provided the original work is properly cited, appropriate credit is given, any changes made indicated, and the use is non-commercial. See: http://creativecommons.org/licenses/by-nc/4.0/.

\section{REFERENCES}

1. Belkaid $Y$, Hand TW. Role of the microbiota in immunity and inflammation. Cell 2014;157:121-41.

2. Marchesi JR, Adams DH, Fava F, et al. The gut microbiota and host health: a new clinical frontier. Gut 2016;65:330-9.

3. Mach N, Fuster-Botella D. Endurance exercise and gut microbiota: a review. J Sport Health Sci 2017;6:179-97.

4. Sanz Y, Olivares M, Moya-Pérez Á, et al. Understanding the role of gut microbiome in metabolic disease risk. Pediatr Res 2015;77:236-44.

5. Manzoor MAP, Rekha PD. Prostate cancer: Microbiome - the 'unforeseen organ'. Nat Rev Urol 2017;14:521-2.

6. Rhee H, Gunter JH, Heathcote P, et al. Adverse effects of androgendeprivation therapy in prostate cancer and their management. BJU Int 2015;115:3-13.

7. Potosky AL, Davis WW, Hoffman RM, et al. Five-year outcomes after prostatectomy or radiotherapy for prostate cancer: the prostate cancer outcomes study. J Natl Cancer Inst 2004;96:1358-67.

8. Sanda MG, Dunn RL, Michalski J, et al. Quality of life and satisfaction with outcome among prostate-cancer survivors. N Engl J Med 2008;358:1250-61.

9. Stensvold A, Dahl AA, Brennhovd B, et al. Bother problems in prostate cancer patients after curative treatment. Urol Oncol 2013;31:1067-78.

10. King MT, Viney R, Smith DP, et al. Survival gains needed to offset persistent adverse treatment effects in localised prostate cancer. $\mathrm{Br} J$ Cancer 2012;106:638-45.

11. Maeda Y, Høyer M, Lundby L, et al. Faecal incontinence following radiotherapy for prostate cancer: a systematic review. Radiother Oncol 2011;98:145-53.

12. Frugé $A D$, Ptacek $T$, Tsuruta $Y$, et al. Dietary changes impact the gut microbe composition in overweight and obese men with 
prostate cancer undergoing radical prostatectomy. J Acad Nutr Diet 2018;118:714-23.

13. Amirian ES, Petrosino JF, Ajami NJ, et al. Potential role of gastrointestinal microbiota composition in prostate cancer risk. Infect Agent Cancer 2013;8:42.

14. Golombos DM, Ayangbesan A, O'Malley P, et al. The role of gut microbiome in the pathogenesis of prostate cancer: a prospective, pilot study. Urology 2018;111:122-8.

15. Cavalieri E, Rogan E. The molecular etiology and prevention of estrogen-initiated cancers: ockham's razor: pluralitas non est ponenda sine necessitate. Plurality should not be posited without necessity. Mol Aspects Med 2014;36:1-55.

16. Harada N, Hanaoka R, Hanada K, et al. Hypogonadism alters cecal and fecal microbiota in male mice. Gut Microbes 2016;7:533-9.

17. Harada N, Hanaoka R, Horiuchi $\mathrm{H}$, et al. Castration influences intestinal microflora and induces abdominal obesity in high-fat dietfed mice. Sci Rep 2016;6:23001.

18. Org E, Mehrabian M, Parks BW, et al. Sex differences and hormonal effects on gut microbiota composition in mice. Gut Microbes 2016;7:313-22

19. Markle JG, Frank DN, Mortin-Toth S, et al. Sex differences in the gut microbiome drive hormone-dependent regulation of autoimmunity. Science 2013:339:1084-8.

20. Klil-Drori AJ, Tascilar K, Yin H, et al. Androgen deprivation therapy and the incidence of inflammatory bowel disease in patients with prostate cancer. Am J Epidemiol 2016;184:15-22.

21. Bressa C, Bailén-Andrino M, Pérez-Santiago J, et al. Differences in gut microbiota profile between women with active lifestyle and sedentary women. PLoS One 2017;12:e0171352.

22. Flint HJ, Duncan SH, Scott KP, et al. Links between diet, gut microbiota composition and gut metabolism. Proc Nutr Soc 2015;74:13-22.

23. Cerdá B, Pérez M, Pérez-Santiago JD, et al. Gut microbiota modification: another piece in the puzzle of the benefits of physical exercise in health? Front Physiol 2016;7:51.

24. Choi Y, Oh DY, Kim TY, et al. Skeletal muscle depletion predicts the prognosis of patients with advanced pancreatic cancer undergoing palliative chemotherapy, independent of body mass index. PLoS One 2015;10:e0139749.

25. Petriz BA, Castro AP, Almeida JA, et al. Exercise induction of gut microbiota modifications in obese, non-obese and hypertensive rats. BMC Genomics 2014;15:511.

26. Mariat D, Firmesse O, Levenez F, et al. The Firmicutes/Bacteroidetes ratio of the human microbiota changes with age. BMC Microbiol 2009;9:123.

27. Ley RE, Turnbaugh PJ, Klein S, et al. Microbial ecology: human gut microbes associated with obesity. Nature 2006;444:1022-3.

28. Smith MR, Finkelstein JS, McGovern FJ, et al. Changes in body composition during androgen deprivation therapy for prostate cancer. J Clin Endocrinol Metab 2002;87:599-603.

29. Galvão DA, Spry NA, Taaffe DR, et al. Changes in muscle, fat and bone mass after 36 weeks of maximal androgen blockade for prostate cancer. BJU Int 2008;102:44-7.

30. Boxer RS, Kenny AM, Dowsett R, et al. The effect of 6 months of androgen deprivation therapy on muscle and fat mass in older men with localized prostate cancer. Aging Male 2005;8:207-12.

31. Clemente JC, Ursell LK, Parfrey LW, et al. The impact of the gut microbiota on human health: an integrative view. Cell 2012;148:1258-70.

32. Villena J, Kitazawa H. Modulation of intestinal TLR4-inflammatory signaling pathways by probiotic microorganisms: lessons learned from lactobacillus jensenii TL2937. Front Immunol 2014;4:512.

33. Kelly RS, Vander Heiden MG, Giovannucci E, et al. Metabolomic biomarkers of prostate cancer: prediction, diagnosis, progression, prognosis, and recurrence. Cancer Epidemiol Biomarkers Prev 2016;25:887-906.

34. Paulsen JA, Ptacek TS, Carter SJ, et al. Gut microbiota composition associated with alterations in cardiorespiratory fitness and psychosocial outcomes among breast cancer survivors. Support Care Cancer 2017;25:1563-70.

35. Chang AJ, Autio KA, Roach M, et al. High-risk prostate cancerclassification and therapy. Nat Rev Clin Oncol 2014;11:308-23.

36. Choo JM, Leong LE, Rogers GB. Sample storage conditions significantly influence faecal microbiome profiles. Sci Rep 2015;5:16350.

37. Al-Ghalith GA, Hillmann B, Ang K, et al. SHI7 Is a self-learning pipeline for multipurpose short-read DNA quality control. mSystems 2018;3.
38. M M. Cutadapt removes adapter sequences from high-thoughput sequencing reads. EMBnetjournal 2011;17:10-12.

39. Callahan BJ, McMurdie PJ, Rosen MJ, et al. DADA2: High-resolution sample inference from Illumina amplicon data. Nat Methods 2016;13:581-3.

40. Cole JR, Wang Q, Fish JA, et al. Ribosomal Database Project: data and tools for high throughput rRNA analysis. Nucleic Acids Res 2014;42:D633-42.

41. Røseth AG, Fagerhol MK, Aadland E, et al. Assessment of the neutrophil dominating protein calprotectin in feces. A methodologic study. Scand J Gastroenterol 1992;27:793-8.

42. Talley NJ, Boyce PM, Owen BK, et al. Initial validation of a bowel symptom questionnaire and measurement of chronic gastrointestinal symptoms in Australians. Aust $N$ Z J Med 1995;25:302-8.

43. Kulich KR, Madisch A, Pacini F, et al. Reliability and validity of the Gastrointestinal Symptom Rating Scale (GSRS) and Quality of Life in Reflux and Dyspepsia (QOLRAD) questionnaire in dyspepsia: a sixcountry study. Health Qual Life Outcomes 2008;6:12.

44. Blake MR, Raker JM, Whelan K. Validity and reliability of the bristol stool form scale in healthy adults and patients with diarrhoeapredominant irritable bowel syndrome. Aliment Pharmacol Ther 2016;44:693-703.

45. Kato J, Nagahara A, lijima K, et al. Evaluation of EORTC QLQ-C30 questionnaire in patients undergoing in-hospital chemotherapy for gastrointestinal cancer in Japan. J Gastroenterol Hepatol 2008;23:S268-72.

46. van Andel G, Bottomley A, Fosså SD, et al. An international field study of the EORTC QLQ-PR25: a questionnaire for assessing the health-related quality of life of patients with prostate cancer. Eur $J$ Cancer 2008;44:2418-24.

47. Hart NH, Nimphius S, Spiteri T, et al. Segmental musculoskeletal examinations using Dual-Energy X-Ray Absorptiometry (DXA): positioning and analysis considerations. J Sports Sci Med 2015;14:620-6.

48. Galvão DA, Spry N, Denham J, et al. A multicentre year-long randomised controlled trial of exercise training targeting physical functioning in men with prostate cancer previously treated with androgen suppression and radiation from TROG 03.04 RADAR. Eur Urol 2014;65:856-64.

49. Galvão DA, Taaffe DR, Cormie P, et al. Efficacy and safety of a modular multi-modal exercise program in prostate cancer patients with bone metastases: a randomized controlled trial. BMC Cancer 2011;11:517.

50. Garnacho-Castaño MV, Domínguez R, Muñoz González A, et al Exercise prescription using the borg rating of perceived exertion to improve fitness. Int J Sports Med 2018;39:115-23.

51. Reinke SN, Galindo-Prieto B, Skotare T, et al. OnPLS-based multiblock data integration: a multivariate approach to interrogating biological interactions in asthma. Anal Chem 2018;90:13400-8.

52. De Roover K, Ceulemans E, Timmerman ME. How to perform multiblock component analysis in practice. Behav Res Methods 2012:44:41-56.

53. Tenenhaus M, Tenenhaus A, Groenen PJF. Regularized generalized canonical correlation analysis: a framework for sequential multiblock component methods. Psychometrika 2017:737-77.

54. Allen JM, Mailing LJ, Cohrs J, et al. Exercise training-induced modification of the gut microbiota persists after microbiota colonization and attenuates the response to chemically-induced colitis in gnotobiotic mice. Gut Microbes 2018;9:115-30

55. Harley IT, Karp CL. Obesity and the gut microbiome: striving for causality. Mol Metab 2012;1:21-31.

56. Focht BC, Lucas AR, Grainger E, et al. Effects of a group-mediated exercise and dietary intervention in the treatment of prostate cancer patients undergoing androgen deprivation therapy: results from the IDEA-P trial. Ann Behav Med 2018;52:412-28.

57. Grosicki GJ, Fielding RA, Lustgarten MS. Gut microbiota contribute to age-related changes in skeletal muscle size, composition, and function: biological basis for a gut-muscle axis. Calcif Tissue Int 2018;102:433-42.

58. Claesson MJ, Jeffery IB, Conde S, et al. Gut microbiota composition correlates with diet and health in the elderly. Nature 2012;488:178-84.

59. van Tongeren SP, Slaets JP, Harmsen HJ, et al. Fecal microbiota composition and frailty. Appl Environ Microbiol 2005;71:6438-42.

60. Costamagna D, Costelli P, Sampaolesi M, et al. Role of inflammation in muscle homeostasis and myogenesis. Mediators Inflamm 2015;2015:1-14 